\title{
Session 21.6: Preserving Dark Skies and Protecting Against Light Pollution in a World Heritage Framework
}

\author{
Malcolm G. Smith \\ NOAO/CTIO, \\ Avenida Juan Cisternas 1600, La Serena, Chile \\ email: msmith@ctio.noao.edu
}

\begin{abstract}
This session opened with a crucial explanation by Michel Cotte of how astronomers first need to understand how to apply UNESCO World Heritage Criteria if they want to motivate their government(s) to make the case to UNESCO for World Heritage recognition. UNESCO World Heritage cannot be obtained just to protect dark skies.

Much more detail of this and the other presentations in this session, along with many images, can be found at the session website: http://www. noao.edu/education/IAUGA2015FM21.

The next speaker, John Hearnshaw, described the Aoraki Mackenzie International Dark Sky Reserve and the work it carries out. This was followed by a wide-ranging summary (by Dan Duriscoe and Nate Ament) of the U.S. National Park Service (NPS) Night Skies Program. The abstract of Cipriano's Marin's paper, "Developing Starlight connections with UNESCO sites through the Biosphere Smart" was shown in his absence. The final presentation (by Arkadiusz Berlicki, S. Kolomanksi and T. Mrozek) discussed the bi-national Izera Dark Sky Park.
\end{abstract}

Keywords. atmospheric effects, site testing

\section{Overview}

As adviser to ICOMOS and IAU, Michel Cotte opened the session by addressing the question of how to apply the UNESCO World Heritage Criteria to the "Windows to the Universe" Sites. He explained the terms of reference issued from the UNESCO World Heritage Convention and the vital details behind establishing the "Justification of the Outstanding Universal Value (OUV) of a given place or site". He then explained how the OUV of a site is expressed by the WH criteria - six of which are possible for cultural value and 4 for natural value. Dark sky is one of the natural attributes giving value to the place (Fig. 1). Unlike all the existing WH sites, sky itself could not be seen as a 'property' in a Human/Juridical (anthropocentric) sense. However, dark-sky quality could also be considered as a cultural attribute in the context of the history of the observatory place. The remarkable atmospheric quality for a given place such as High Mountain Observatories explains their settlement; their foundation as "Windows to the Universe" is partly justified by the dark sky quality. The human presence of the observers and astronomers gives meaning and life to the place, supporting important additional intangible value from its history of knowledge and human representations. The dark sky quality can be presented as a local environmental attribute. Studying a place from a World Heritage perspective starts by an inventory of its tangible attributes. Generally speaking, as mentioned earlier, a given place has a series of natural attributes and cultural attributes supporting and expressing its value. Clearly, intrinsic dark sky quality is a natural attribute of the place, among others. A discussion of dark sky quality must include an analysis of its properties (purity as a minimum of physical constraints). Visible 

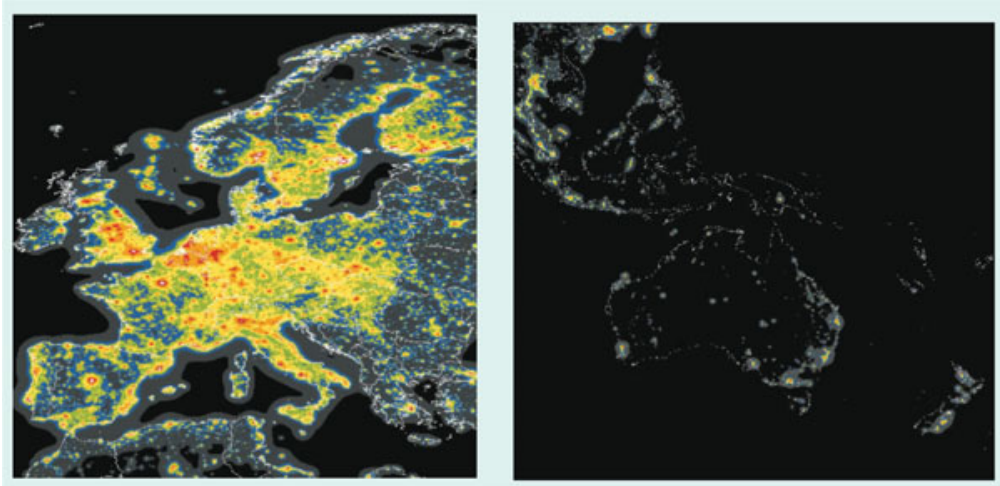

Figure 1: Dark Sky as one of the natural attributes giving value to the "Windows to the Universe" site

relationships between attributes give a landscape value. One can think of dark sky and the atmosphere below it as the "glass" in a "Window to the Universe", i.e. one of the physical attributes giving a specific value to the site. The site itself is then the "Frame" of the window and it has relevant properties in a WH context such as geography, atmosphere, architecture, landscape and nature. Turning to its history, the "Window" has come to be used by humankind with artifacts and instruments.

Thus, in presenting and discussing the OUV of individual sites in a World Heritage context in this session, Michel Cotte reminded us of the need to present Dark Skies among a cluster of natural attributes. We need to consider dark skies as a component of the global natural context of a given place. It belongs to a larger group of natural attributes of the site, forming its natural environmental components. One of the best ways to use Dark-Sky Value is among an ensemble of other remarkable attributes which all combine to constitute a generally remarkable landscape, remarkable at night and remarkable in daylight. Potential OUV is what can result from such a combination of remarkable attributes. Significance and beauty of the whole could be largely the result of the simple addition of individual attributes - which give a landscape specific value. World Heritage Criterion (vii) could be one to concentrate on - a monument of nature.

He also added the wisdom of including Dark Sky management for scientific efficiency and heritage preservation. Points to include are the international success and the importance of the Dark Sky reserves initiatives. Maintenance of the dark-sky quality could be presented as a strong management goal for an astronomical site and region. This leads naturally to the concepts of Dark Sky Reserve and WH Buffer Zones. Detailed examples of such places in different parts of the world provided the content for the rest of this Session.

\section{Outstanding Sites \& Evolution of Protection}

John Hearnshaw spoke next and described the Aoraki Mackenzie International Dark Sky Reserve and light-pollution issues in New Zealand - and how the reserve is managed and promoted to the public to make them aware of light pollution issues and in order to promote star-gazing and astro-tourism. His full presentation can be found at http: //www.noao.edu/education/files/IAU_AMIDSR_Hawaii_2015_short.pdf.

New Zealand has consistently been one of the world leaders. In 1981, a Lighting Ordinance was drawn up in the Mackenzie District Plan. It was enacted through the Town and Country Planning Act 1977. The Plan controls outdoor lighting, specifying types of light, full cut-off, limits emission below $440 \mathrm{~nm}$, restricts times when outdoor recreational 
illumination is permitted. The objective of the ordinance is "Maintenance of the ability to undertake effective research at the Mt. John University Observatory and of the ability to view the quality of the night sky'. The lighting ordinance applies over a large area of the MacKenzie Basin, including all of Lakes Tekapo and Pukaki; this area stretches $60 \mathrm{~km}$ EW and $100 \mathrm{~km}$ NS.

John went on to discuss clearly why a Dark Sky Reserve was needed, given the existence of a lighting ordinance for over 30 years. He gave the following answer: (1) A dark-sky reserve is about (a) marketing and branding; (b) education (c) inspiration. (2) A reserve helps promote the romance of astronomy and allows people to connect spiritually with the universe (3) It also reinforces the message on the need to control light pollution.

These aims are very different from those of the lighting ordinance, which is strictly a legal document to protect the night sky, mainly for astronomical research, with public stargazing as a secondary aim, and the development of astro-tourism is not mentioned at all.

AMIDSR is the world's largest IDA International Dark Sky Reserve $\left(4,367 \mathrm{~km}^{2}\right)$ and the first to obtain gold-tier status (recognized by IDA in June, 2012, following the application made in January of that year). It was the first IDSR in the southern hemisphere (Fig. 2). Views from space (Cinzano et al., Wold Atlas of Artificial Night Sky Brightness, 2001) demonstrate the dramatic contrast between the light (and wasted energy) going up into space from Europe versus that from New Zealand, particularly the western part of the South Island (Fig. 4). Viewed from Mt. John, there is still some light pollution from Tekapo, but the situation is generally good. Tekapo skies are still very dark.

John then mentioned that the World Heritage convention held in Christchurch in 2007 marked the beginning of a campaign to lobby for WH recognition of the dark sky above Mackenzie Basin. At the time this seemed reasonable, given that the Basin adjoins the WH site Te Wahipounamu (which was inscribed in 1990) (Fig. 3). It is an area of magnificent primeval vistas: snow-capped mountains, glaciers, forests, tussock grasslands, lake, rivers, wetlands and over $1,000 \mathrm{~km}$ of wilderness coastline with OUV. His group completed short and full thematic case studies on the Aoraki Mackenzie site for the IAU-ICOMOS International WG on Astronomy and World Heritage.

As mentioned earlier by Michel Cotte, World Heritage Criteria require OUV (Outstanding Universal Value). John Hearnshaw recognized that two of the WH criteria for natural sites may be relevant to the Mackenzie Basin in New Zealand.

(vii) to contain superlative natural phenomena or areas of exceptional natural beauty and aesthetic importance;

(viii) to be outstanding examples representing major stages of earth's history, including the record of life, significant on-going geological processes in the development of landforms or significant geomorphologic or physiographic features.

In recent years it has become clear to John and to many others that an outstanding night sky free of light pollution is not recognized as OUV by the WHC, and is unlikely to be any time soon. John outlined New Zealand's rather disappointing record - it has only three WH sites and only one in he South Island. It already has 8 natural sites on its tentative list for consideration as WH sites, but there has been no movement on this list in the last 8 years.

As hinted above by John, a possible strategy is to apply for an extension of the adjoining Te Wahipounamu WH site so as to include the Mackenzie Basin, citing criteria (vii) and (viii).

Dan Duriscoe and Nate Ament authored the next presentation covering a huge effort aimed at Night Sky Protection and Restoration in U.S. National Parks. This has come such a long way from early, small attempts from Hawaii that was witnessed in the 


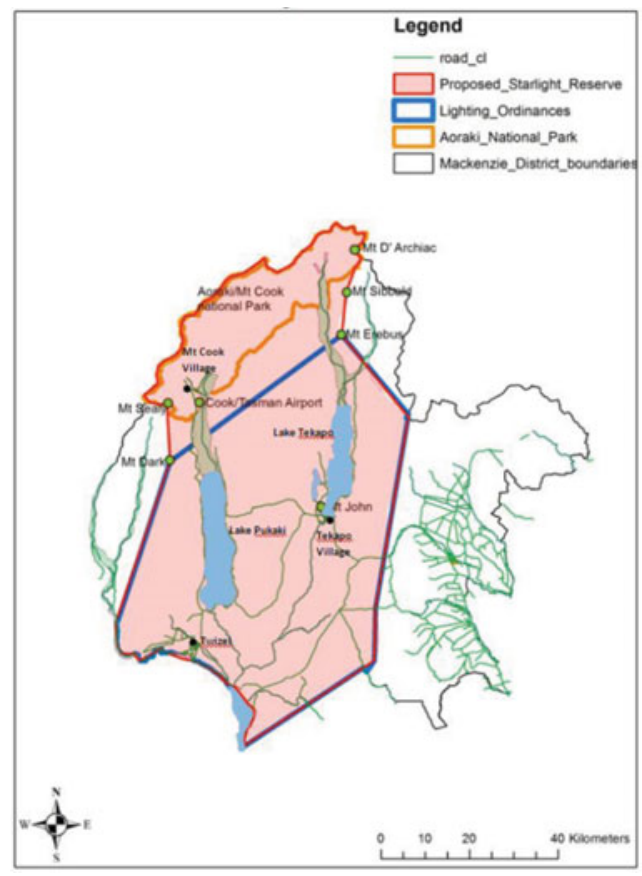

Figure 2: The Aoraki Mackenzie International Dark Sky Reserve, recognized by IDA in June 2012 as the first IDSR in the southern hemisphere.

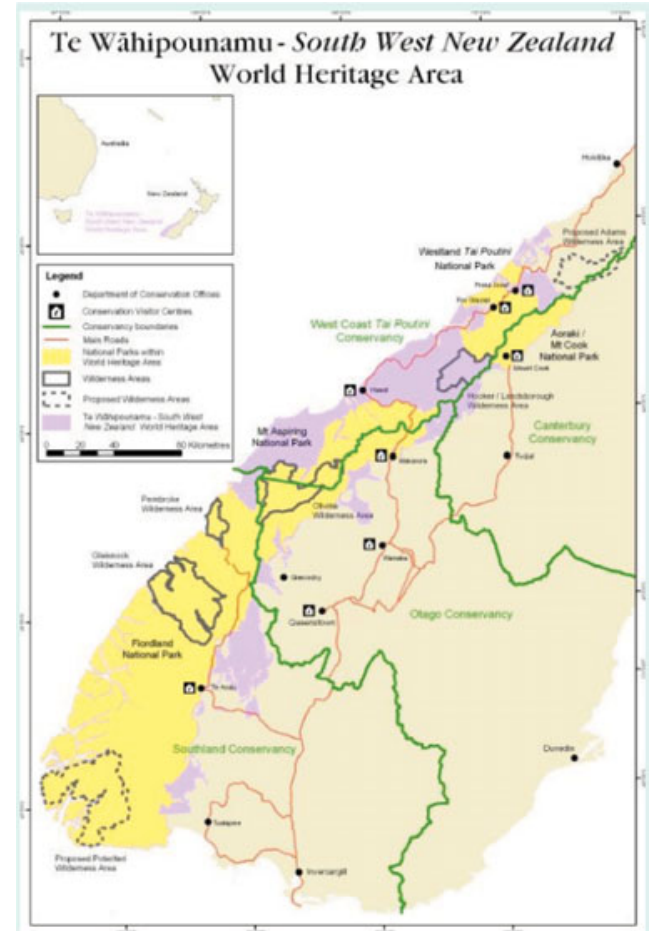

Figure 3: Te Wahi Pounamu, the South Island of New Zealnd's only World Heritage site, has 2.6 million hectares of OUV wilderness and natural beauty.
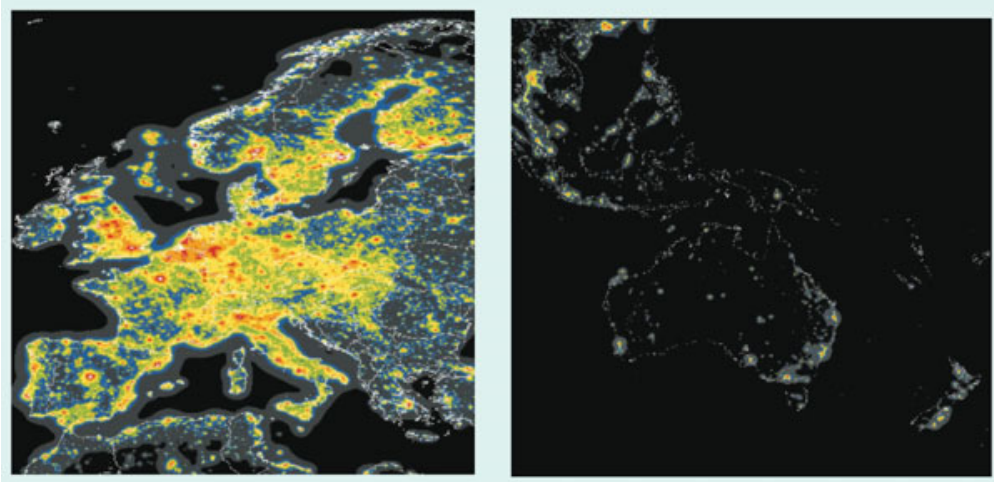

Figure 4: The light recorded in these satellite images represents wasted light and wasted energy going up into space. Image from Cinzano et al., 2001. 


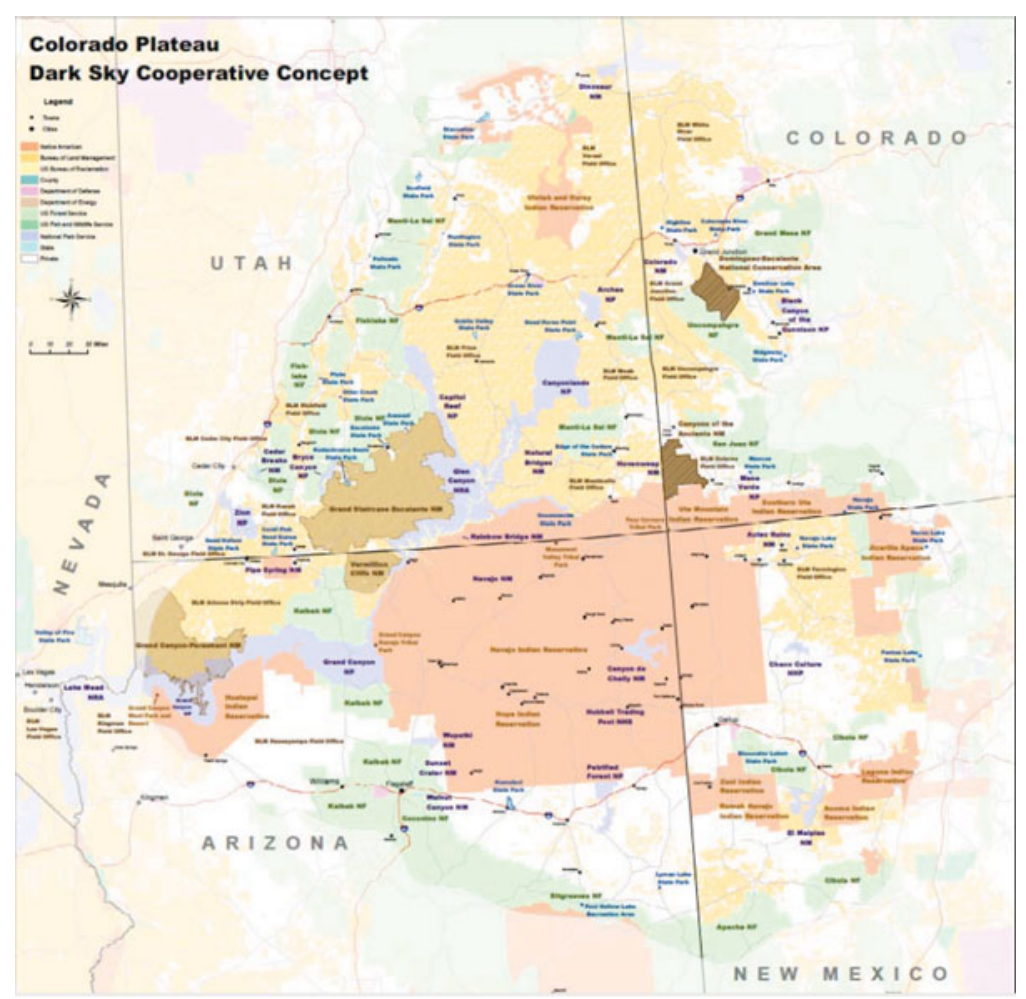

Figure 5: The Colorado Plateau Dark Sky Cooperative Concept

1980s - which were rejected by Washington as irrelevant to the mission of the NPS. The NPS now has a truly remarkable record of leadership in action, measurement, documentation, education and of course outreach on a huge, yet balanced scale that is an example to the world. (See http://www.noao.edu/education/IAUGA2015FM21/ IAUGA2015FM21/2-6-04.)

Dan and Nate describe how the U.S. National Park Service (NPS) Night Skies Program contributes to the recognition of certain outstanding NPS lands as dark sky places. A combination of efforts including measuring resource condition, within-park outdoor lighting control, education outreach for visitors, and engagement with surrounding communities helps establish and maintain such places. In certain circumstances, communities and protected areas join forces in a cooperative effort to preserve the natural nocturnal environment of a region. One recent example, the Colorado Plateau Dark Sky Cooperative, is taking lighting, conservation, and educational steps to fulfill the mission of the NPS Call To Action- Starry Starry Night. This voluntary initiative forms America's first Dark Sky Cooperative, and links communities, tribes, businesses, state/federal agencies, and citizens in a collaborative effort to celebrate the view of the cosmos, minimize the impact of outdoor lighting, and ultimately restore natural darkness to the area. The authors presented progress and accomplishments of established dark sky parks and reserves in the western U.S., with particular emphasis on public response to the actions taken and the results achieved.

The presentation opened with a description of the "Colorado Plateau Dark Sky Cooperative", accompanied with associated images (Figure 5). The Colorado Plateau shows up clearly in images from space provided by the NPS Natural Sounds and Night Skies 


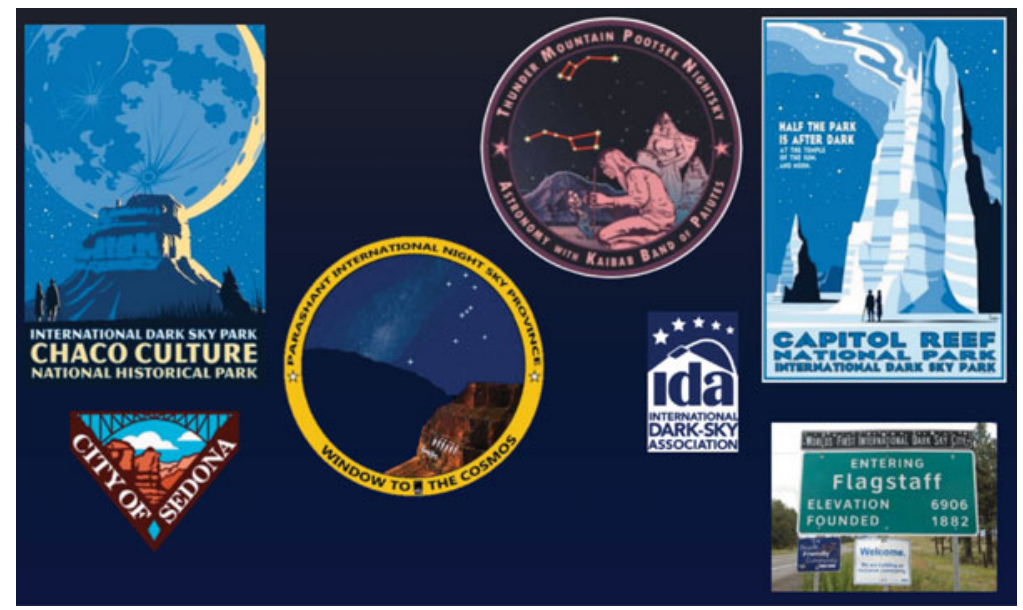

Figure 6: International Dark Sky Parks/Places involved in the "Colorado Plateau Dark Sky Cooperative"

Division as one of the darkest areas of the continental United States. This Co-operative has the advantages of access to high elevation, low precipitation, a large number of clear days, clear, unpolluted air, low population density, dramatic landscape, worldwide visibility and a large amount of public land. Their presentation shows a map of the Plateau showing the Co-operative's involvement in regular stargazing sites and IDA Certified Dark Sky Places. For more detail search "Clear Air and Magnificent Skies (Utah)" and "Grand Canyon In Depth Night Sky". International Dark Sky Parks/Places include the Chaco Culture National Historical Park, Thunder Mountain Pootsee Nightsky - Astronomy with Kaibab Band of Paiutes, Capitol Reef National Park (an International Dark Sky Park), Flagstaff - the world's first international dark sky city, Parashant International Night Sky Province and the City of Sedona (Figure 6).

On the education side, there is an NPS booklet - Junior Ranger Night Explorer serving as an explorer's activeity guide for ages 5-12. In 2014 Bryce Canyon Night Sky Sanctuary led the number of night-sky-related park contacts (approx. 30,000). Others included Parashant (656), Black Canyon (6,356) Canyonlands (761) and Capitol Reef (3,766).

Regarding astrotourism and economic benefits, for Bryce Canyon (2012) astronomyrelated attendance accounted for over 50,000 visits and US\$2 million contributed to local economies. Tourism office campaigns have increased some park visitation by up to 30 percent. Regarding the importance of dark skies to NPS visitors, numbers indicating that Dark Skies are "Important" or "Very Important" over the period from the early 1990's to the late 2000's have increased five-fold. Spending per visiting party has increased from $\$ 90$ to $\$ 270-390$ (day vs. overnight). This change represents an additional $\$ 1.68$ billion value added to Colorado Plateau economies over 10 years. The number of visitors has increased in the off-peak seasons and provide a longer, more sustained period of tourism activity.

In the area of civic engagement and partnerships, Flagstaff is the world's first International Dark Sky City; it has set standards and examples for comprehensive night-sky friendly lighting. Sedona has achieved a recent IDSC designation. Work is in progress with Moab, Monticello, Springdale and others.

Regarding plans for the future, these include extendion of the following current activities: (1) Develop Lightscape Management plans for Parks. (2) Inventory lights and plan for retrofits.(3) Work with partners to collect night sky quality data. (4) Collaborate 
with interpreters to develop night-sky resource educational materials (5) Engage with gateway communities and local businesses (6) Pursue International Dark Sky Park or similar designation.

Measurements of success include: (1) number of light fixtures retrofitted to be nightsky friendly (2) number of parks/communities designated as International Dark Sky Places. (3) number of guided tours that incorporate night-sky education and conservation (rafting trips, expeditions, OUV tours, astro tours). The website for this Focus Meeting Session contains the power points for most of these talk, including this one which presents a "Colorado Plateau Dark Sky Co-operative Measurements of Success'tracking spreadsheet.

Success stories include (1) Created night skies curriculum and training for Colorado Plateau outdoor guides and outfitters, reaching an estimated 40,000-50,000 trip participants each year. (2) 2014 - Partnered with Lowell Observatory and the Keystone Center to host the first Dark Skies and Emerging Technologies conference. (For me this transdisciplinary conference represented the first serious effort I have seen to start to engage all parties involved in, or affected by, the lighting of our planet). (3) Since 2013, 138 new media articles and videos featuring Colorado Plateau dark skies and the Cooperative. (4) 4 newInternational Dark Sky Parks since 2013 (PARA, CHCU, HOVE, CARE). (5) 3 new International Dark Sky Communities. (6) 9 National Parks, 3 State Parks and 3 communities are pending.

The slogan "Half the Park is after Dark" leaves a memorable impression as did the NPS-related presentation.

Cipriano Marin submitted the following abstract found at http://www.noao.edu/ education/IAUGA2015FM21/2-6-05, "Developing Starlight connections with UNESCO sites through the Biosphere Smart". He pointed out that the large number of UNESCO Sites around the world, in outstanding places ranging from small islands to cities, makes it possible to build and share a comprehensive knowledge base on good practices and policies on the preservation of the night skies consistent with the protection of the associated scientific, natural and cultural values. In this context, the Starlight Initiative and other organizations such as IDA play a catalytic role in an essential international process to promote comprehensive, holistic approaches for dark sky preservation, astronomical observation, environmental protection, responsible lighting, sustainable energy, climate change and global sustainability.

Many of these places have the potential to become models of excellence to foster the recovery of the dark skies and its defence against light pollution, including some case studies mentioned in the Portal to the Heritage of Astronomy. Fighting light pollution and recovering starry sky are already elements of a new emerging culture in biosphere reserves and world heritage sites committed to acting on climate change and sustainable development. Over thirty territories, including biosphere reserves and world heritage sites, have developed successful initiatives to ensure night sky quality and promote sustainable lighting. Clear night skies also provide sustainable income opportunities as tourists and visitors are eagerly looking for sites with impressive night skies.

Taking into account the high visibility and the ability of UNESCO sites to replicate network experiences, the Starlight Initiative has launched an action In cooperation with Biosphere Smart, aimed at promoting the Benchmark sites. Biosphere Smart is a global observatory created in partnership with UNESCO MaB Programme to share good practices, and experiences among UNESCO sites. The Benchmark sites window allows access to all the information of the most relevant astronomical heritage sites, dark sky protected areas and other places committed to the preservation of the values associated with the 


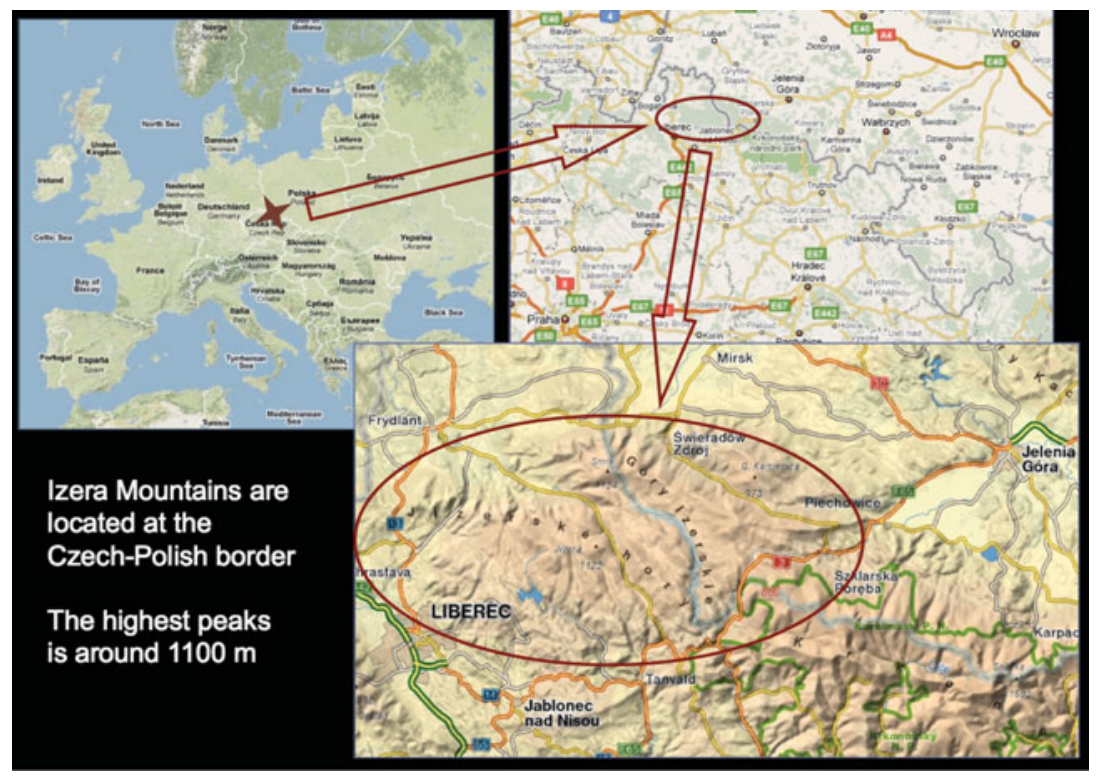

Figure 7: The location of the Izera Dark Sky Park in Europe and at the Czech-Polish border.

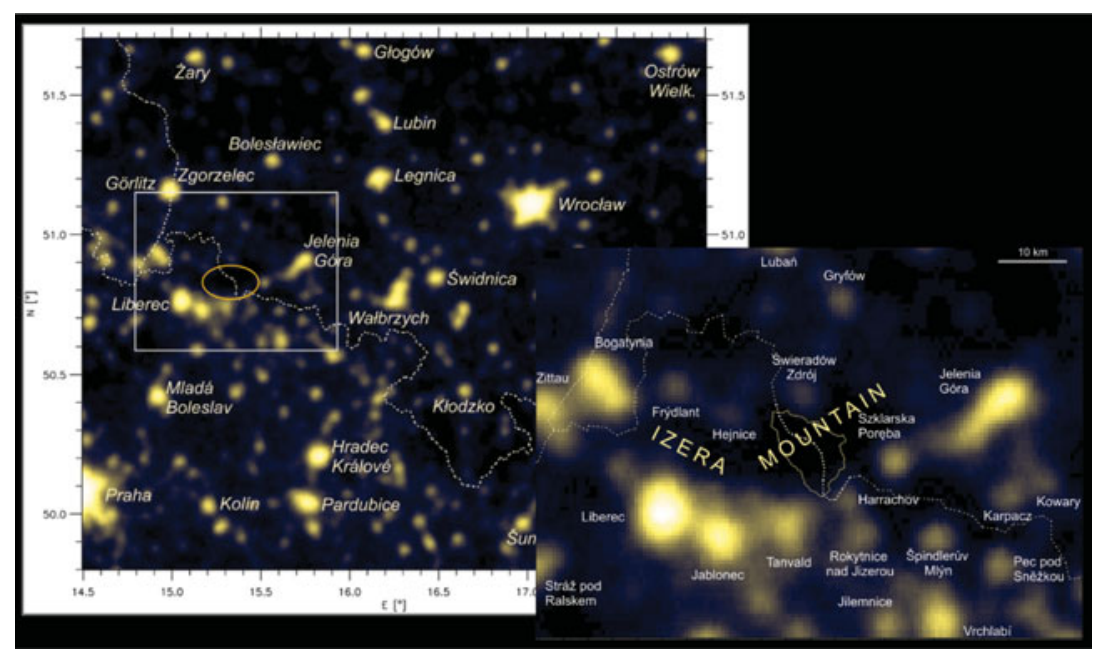

Figure 8: Southwest Poland/North Czech Republic at night - images are from the Defense Meteorological Satellite Program.

night sky. This is a new step ahead in our common task of protecting the starry skies at UNESCO sites.

The final presentation in the session was by Arkadiusz Berlicki, S. Kolomanksi and T. Mrozek (http://www .noao.edu/education/IAUGA2015FM21/2-6-07), who discussed dark-sky protection and education enabled at the Izera Dark-Sky Park (Figures 7 and 8). Their presentation emphasized that darkness of the night sky is a natural component of our environment which should be protected against negative effects of human activities. Darkness at night is necessary for balanced life of plants, animals and people. Unfortunately, development of human civilization and technology has led to substantial 


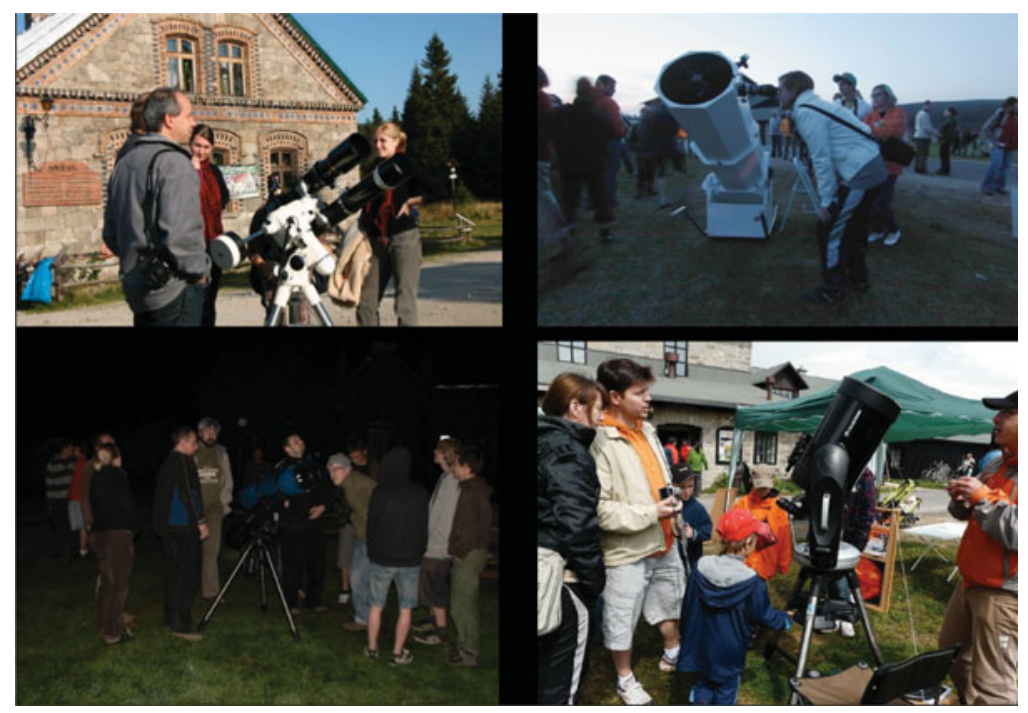

Figure 9: Astronomical events for the public: Polish-Czech Astronomical Days, amateur astronomy meetings, etc

increase of the night-sky brightness and to a situation where nights are no longer dark in many areas of the world. This phenomenon - light pollution - can be ranked among such problems as chemical pollution of air, water and soil. Besides the environment, the light pollution can also affect e.g. the scientific activities of astronomers - many observatories built in the past began to find themselves within the glow of city lights making their (night-time) observations difficult, or even impossible. In order to protect the natural darkness of nights many so-called "dark sky parks" were established, where the darkness is preserved, similar to typical nature reserves. The role of these parks is not only conservation but also education, supporting the effort to make society aware of how serious the problem of the light pollution is.

The history of dark sky areas in Europe was initiated on November 4, 2009 in Jizerka, a small village situated in the Izera Mountains, when Izera Dark Sky Park (IDSP) was established. It was the first international, trans-boundary dark sky park in the world. The idea of establishing that dark sky park in the Izera Mountains originated from a need to give to the society in Poland and the Czech Republic knowledge about light pollution. Izera Dark Sky Park is a component of the astro-tourism project, "Astro Izery", that combines the tourist attraction of Izera Valley and astronomical education under the wonderful starry Izera sky. Besides the IDSP, the project, Astro Izery, consists of the set of simple astronomical instruments (gnomon, sundial), a naturel trail "Solar System Model", and astronomical events for the public (Figure 9). In addition, twice a year they organize a 3-4 day "Astronomy Workshop for Schools", where teachers and astronomers from the Astronomical Institute (University of Wroclaw) educate the young generations in the field of astronomy and other physical sciences.

\section{Path Forward}

Initiatives such as the above and continued networking between these and other similar groups will continue to provide ideas for protection of starlit sites across the world. They may also provide their governments with options for to explore further action with 
UNESCO separately or together in the form of one or more serial nominations. It is becoming clear, from these joint sessions between FM2 and FM21, that the Chilean government is becoming increasingly interested in working with UNESCO and the IAU on these issues, as they apply to the optical, mountain-top observatories in northern Chile. 